\title{
Generation of Induced Pluripotent Stem Cells from Conjunctiva
}

\author{
Jin Yang, MD, PHD ${ }^{1,2}$, Yao Li, MD², Deniz Erol' ${ }^{2}$, Wen-Hsuan $\mathrm{Wu}^{2}$, Yi-Ting Tsai ${ }^{2}$, Xiao-Rong \\ Li, MD, PHD ${ }^{1}$, Richard J. Davis, PHD², and Stephen H. Tsang, MD, PHD ${ }^{2}$ \\ Jin Yang: yangjinchina324@gmail.com; Yao Li: yl2635@columbia.edu; Deniz Erol: dze2101@columbia.edu; Wen-Hsuan \\ Wu: ww2303@columbia.edu; Yi-Ting Tsai: yt2335@columbia.edu; Xiao-Rong Li: xiaorli@163.com; Richard J. Davis: \\ rd2224@columbia.edu; Stephen H. Tsang: sht2@columbia.edu \\ ${ }^{1}$ TianJin Medical University Eye Hospital, TianJin, 300384, China \\ ${ }^{2}$ Bernard and Shirlee Brown Glaucoma Laboratory, Edward S. Harkness Eye Institute, Columbia \\ University Medical Center, New York, NY 10032, USA
}

\section{Abstract \\ Purpose-The objective of this study was to determine whether cells from the conjunctiva could be reprogrammed into induced pluripotent stem (iPS) cells, providing an alternative source of stem cells.}

\begin{abstract}
Methods-We employed a doxycycline induced reprogrammable mouse strain to generate iPS cells from conjunctiva. The identity of the stem cells was confirmed by Reverse transcription polymerase chain reaction (RT-PCR) and immunofluorescence assays. Immunocytochemistry and teratoma assays are established means for scoring stem cell pluripotency. The reprogramming efficiencies of conjunctiva cells and ear fibroblasts were compared.

Results-We confirmed the identity of the stem cells and demonstrated expression of pluripotency markers (OCT4, SOX2, NANOG, and SSEA1), as tested by RT-PCR and immunofluorescence assays. In addition, derived iPS cells differentiated successfully into embryoid bodies and showed teratoma formation when injected into immunodeficient mice. Reprogramming conjunctival tissue is as efficient as reprogramming ear fibroblasts. ConjunctivaiPS exhibited classic features of embryonic stem (ES) cells with respect to morphology, expression of surface antigens and pluripotency-associated transcription factors, capacity to differentiate in vitro, and the ability to form all three germ layers in vivo.
\end{abstract}

Conclusion-The present study demonstrated that conjunctival cells, which are readily obtained during the course of many routine conjunctival biopsies and ophthalmic procedures, can be another reliable source of iPS cells.

\section{Keywords}

conjunctiva; induced pluripotent stem cells; reprogramming; cells cultured

\section{Introduction}

Induced pluripotent stem (iPS) cells are a useful alternative to embryonic stem (ES) cells as a source for stem cell-based therapies. These cells, derived from patients' own tissues, are expected to become a powerful tool in the arsenal of regenerative medicine. Not only have

\footnotetext{
Corresponding author: Stephen H. Tsang. MD, PhD, Edward Harkness Eye Institute, 160 Fort Washington Ave, Research Annex, Room 513, New York, NY 10032, Phone: 212-342-1189, Fax: 212-305-4987, sht2@ columbia.edu.

Conflicts of Interest and Source of Funding:

Competing Interests: The authors have no proprietary or commercial interests in any concept or product discussed in this article.
} 
iPS cells been the object of fewer ethical controversies than ES cells, but autologous iPSbased transplants would also carry medical advantages by eliminating the pitfalls and risks of immunosuppressive therapy. Already, previous research has shown promise in rescuing degenerated retinal pigment epithelium (RPE) cells with iPS treatment in mouse models of retinal disease [1].

Previous studies have shown it is possible to generate these multipurpose, pluripotent stem cells from various body tissues, including stomach cells [2], liver cells [2], pancreatic B cells [3], lymphocytes [4], neural progenitor cells [5,6], and human keratinocytes [7,8]. Many of these experiments employed genetic labeling or other techniques to confirm the identity of donor cells. Due to the difficulty of sampling liver cells during regular physical examinations and uncertainty whether hair cells and adipose cells are a reliable source for reprogramming $[9,10]$, the search for an optimal tissue source for iPS has continued. Skin cells are still the most predominant and commonly used source of iPS. However, routine ophthalmic procedures offer numerous opportunities to take samples of other tissues, such as the conjunctiva [11].

The conjunctiva is a thin, transparent mucous membrane that lines the posterior surface of the lid and is reflected forwards on the eye. Many ophthalmic procedures involve conjunctival biopsies, either at the diagnosis or intervention stages [11]. If conjunctival tissue is indeed reprogrammable, these biopsies could be stored and used to generate iPS at a later date. There would be no need for a subset of patients to undergo additional skin biopsies. Never before investigated as a source of iPS cells, conjunctival tissue was tested in this study to determine its availability for reprogramming.

\section{Materials and Methods}

\section{Mice}

Procedures were approved by the Institutional Animal Care and Use Committee of Columbia University (protocol \#AC-AAAB4306). Treatment of mice adhered to the Statement for the Use of Animals issued by the Association for Research in Vision and Ophthalmology and the Policy for the Use of Animals in Neuroscience Research issued by the Society for Neuroscience. Mice employed in the study were single-gene transgenic $R 26^{r t T A}$; Collal ${ }^{4 F 2 A}$ mice (Jackson Laboratories) expressing a dox-inducible polycistronic $4 \mathrm{~F} 2 \mathrm{~A}$ cassette containing the four reprogramming genes Oct4, Sox2, Klf4, and Myc from the Collal locus. Somatic expression of these reprogramming factors allows multiple somatic cell types to be directly reprogrammed to generate induced pluripotent stem cells by culture with doxycycline.

\section{Conjunctiva Primary Cell Culture}

Under sterile conditions, mice were anesthetized by intraperitoneal injection with a combination of $1 \%$ xylazine and $10 \%$ ketamine. Eyelashes were excised from right eyes, and then both of eyelids and ocular surface were cleaned with a 5\% Povidone Iodine solution and rinsed with sterile phosphate buffered saline (PBS). Subconjunctival injections of $0.2 \mathrm{ml}$ PBS were administered to separate conjunctival tissue, and small samples $(2 \mathrm{~mm} \times$ $3 \mathrm{~mm}$ ) were obtained by biopsy from the upper temporal conjunctival fornix. The conjunctiva biopsy tissue was next washed in PBS three times before dissection into explants of $1 \mathrm{~mm} \times 2 \mathrm{~mm}$ in size. Tissues were then cultured in DMEM (Dulbecco's modified Eagles's medium) supplemented with high glucose, $10 \%$ fetal bovine serum, antibiotic (50 U/mL penicillin, $50 \mu \mathrm{g} / \mathrm{mL}$ streptomycin) and Sodium Pyruvate (all from Invitrogen, Carlsbad, CA). Tissue was maintained in $1 \mathrm{~mL}$ tissue-culture medium for a period of 6 hours until cells were attached closely to culture dishes. Then an additional $3 \mathrm{~mL}$ 
of the tissue-culture medium was added. After initial outgrowths appeared at day 7 , conjunctiva cells were collected and purified for iPS cell induction. Primary conjunctival cell cultures were typically intermixed with fibroblast cells, since there are several layers of conjunctival tissue. $1 \mathrm{mg} / \mathrm{ml}$ collagenase IV was administered to primary conjunctival cells for about 30-40 minutes. The majority of conjunctival fibroblasts detached earlier than epithelial cells and were separated from epithelial cells. The majority of resulting cell sheets consisted of epithelium cells [12]. Keratin 13 (K13) and cytokeratin 15 (CK15) are both specific markers of conjunctival epithelium $[13,14]$. To confirm that these conjunctival monolayer cells were indeed include conjunctival epithelium cells, a K13 RT-PCR assay was performed. CK 15 immunofluorescence staining was also performed before and after collagenase IV purification to show the proportion of conjunctival cells that are epithelial or fibroblast in origin. The total numbers of cells were identified by immunolocalization with vimentin, which is expressed both on epithelium cells and fibroblasts [14, 15].

Mouse ear fibroblast cell culture-Mouse ear tissue tips were collected after cleaning with a 5\% Povidone Iodine solution, and were subsequently rinsed with sterile PBS. These were cut into small pieces and seeded on a dish with a cover slip to help tissue attach to the dish. A small amount of silicon oil was placed at the center of the plates to help them stick to cover slips. After initial outgrowths appeared at day 7, the cover slip was turned over and $0.25 \%$ trypsin was added for 3 minutes. The medium consisting of $10 \%$ serum to stop trypsinization was added. Fibroblast cells were fully detached from the dish with a cell scraper. After cells were centrifuged, cell pallets were suspended; within one minute, large pieces settled to the bottom. The suspended cells were then reseeded on a dish.

\section{iPS Cell Induction}

Primary cultured conjunctival cells were seeded at a $2.5 \times 10^{5}$ concentration per $10 \mathrm{~cm}$ dish for reprogramming and colony isolation. Medium was replaced with iPS medium fresh prepared and supplemented with doxycycline (dox) to a final concentration of $2 \mu \mathrm{g} / \mathrm{ml}$. We also added medium without dox as a negative control. Dishes were incubated at $37^{\circ} \mathrm{C}$ in a $5 \% \mathrm{CO}_{2}$ incubator. Dox was withdrawn at day 12. For efficiency comparisons, a total of 8 dishes were created, derived from 2 separate genetically engineered mice. Four dishes of conjunctival cells and four dishes of ear fibroblast cells were derived from each of these mice. They were seeded at $1.4 \times 10^{5}$ concentrations per $3.5 \mathrm{~cm}$ dish.

Mouse iPS culture medium was composed of mouse embryonic stem cells (mES) medium, $10 \%$ ES cell Grade FBS, $0.1 \mathrm{mmol} / \mathrm{L}$ nonessential amino acids, $1 \mathrm{mmol} / \mathrm{L}$ Glutamax, 0.1 $\mathrm{mmol} / \mathrm{L} \beta$-mercaptoethanol, $4 \mathrm{ng} / \mathrm{ml}$ of basic fibroblast growth factor (bFGF) (all from Invitrogen, Carlsbad, CA), and 1,000 units/mL leukemia inhibitory factor (LIF) (Millipore, Billerica, MA). Cells were cultured at $37^{\circ} \mathrm{C}$ in a $5 \% \mathrm{CO}_{2}$ incubator. At day 20 , colonies formed. Ideal colonies on $10 \mathrm{~cm}$ dishes were micro-dissected into small piceses using glass hooks. This process was repeated until passage 3 , at which time colonies were picked manually and incubated in $0.25 \%$ trypsin (Invitrogen) for 20 minutes at $37^{\circ} \mathrm{C}$. Then colonies were dissociated into single cells and transferred to wells in 96-well plates with mouse embryonic fibroblast (MEF) feeder cells and $200 \mu \mathrm{L}$ medium. The iPS cells were further expanded into six-well plates. After expansion to six-well plates, the cells were routinely split at a 1:6 ratio every 2-3 days. The colonies in comparison groups, in the $3.5 \mathrm{~cm}$ dishes, were not picked. They were cultured for alkaline phosphatase (AP) staining, which was performed in each separate $3.5 \mathrm{~cm}$ dish on days 20 and 25. Induction ratios of iPS cells in the conjunctival cell and ear fibroblast dishes were compared. The numbers of AP positive staining colonies were counted. 


\section{Embryonic Body Formation and Teratoma Formation}

After iPS had formed, the mES medium was switched to one without LIF and bFGF. Cells were cultured for another 10 days. After 10 days, embryoid bodies appeared. Conjunctivaderived iPS cells differentiated from the two reprogrammed mice were subcutaneously injected into 6 Prkd $c^{\text {scid } / P r k d} c^{\text {scid }}$ mice (Jackson Laboratories). After five weeks, teratomas generated from the outgrowth of iPS were analyzed by H\&E staining.

\section{Immunofluorescence Staining}

Both ES cells with the C57BL6/J mouse background and two conjunctiva-iPS cell lines were fixed in 4\% paraformaldehyde (Sigma) in PBS for 2 hours at room temperature. AntiSSEA1, anti-SOX2, and anti-OCT4 immunofluorescence staining was performed with a mouse ES/iPS cell characterization kit (Applied StemCell). Alkaline phosphatase (AP) was tested. AP is a stem cell membrane marker whose elevated expression is associated with pluripotency [16]. This assay has been widely used to examine the differentiated/ undifferentiated status of ES/iPS cells.

\section{RT-PCR Assay}

RNA was isolated from conjunctiva-derived cells, two iPS cell lines and embryonic stem cells using the RNeasy kit (QIAGEN, Hilden, Germany), and genomic DNA contamination was removed using a DNA-free kit (Invitrogen). One microgram of total RNA was used for cDNA synthesis using Superscript III Reverse Transcriptase and Oligo (dT) primers (Invitrogen). PCR was performed using primers listed in Table 1.

\section{Karyotyping}

Two iPS cell lines were arrested in metaphase by the addition of colcemid $(5 \mathrm{ul} / \mathrm{ml}$, Invitrogen) to the culture medium and treated for 2 hours. $0.56 \% \mathrm{KCl}$ solution was added after centrifuge and cells were carefully resuspended. Cells were then fixed with ice-cold 3:1 methanol: glacial acetic acid. A single drop of cell suspension was released onto clean glass microscope slides (cleaned with 70\% ethanol) from approximately $40 \mathrm{~cm}$ height, blown across the surface and allowed to air dry. This was stained with Giemsa solution and put under a coverslip. Chromosomes were counted.

\section{Results}

Conjunctiva cells began growing out of a biopsy taken from $R 26^{r t T A}$; Colla1 ${ }^{4 F 2 A}$ mice starting at day 2 (Figure 1). $30 \%$ of cells derived from the conjunctiva expressed the CK 15 epithelial marker at passage 1(P1) (Figure 2). The proportion of CK 15 reactive cells increases after collagenase IV treatment and replating at P2 (Figure 2). iPS differentiation was initiated with doxycycline (Dox) at P2. After three days of dox treatment, the dox+ the cells began to change shape and assume the characteristic dome-shaped, refractile colonies of iPS cells (Figure 3B); in contrast, no changes were apparent in a control untreated group except for cell growth (Figure 3A). On day 20, ES-like colonies had formed. Colonies in 10 $\mathrm{cm}$ dishes were picked for further passage (Figure 4A). These cells have been maintained for $>10$ passages (Figure 4C). After passages were manually picked, the cells formed compact colonies displaying a peripheral halo when observed by phase-contrast microscopy, closely resembling the formations commonly observed in mouse ES cell colonies (Figure 4F). The numbers of colonies per $3.5 \mathrm{~cm}$ dish were counted, both in conjunctival cell lines and ear fibroblast cell lines, on day 20 and day 25, after AP staining (Figure 5). In some cases, colonies that had formed by days 20 or 21 and were maintained in culture without subculturing became differentiated over the next 4-5 days. When those iPS colonies were stained on day 25 , these were not stainable. While new colonies which continued to appear 
during these 5 days were stainable. The numbers of colonies increased dramatically and were present at similar levels in both iPS lines.

Immunofluorescence analysis showed that the iPS cell lines stained positively for OCT4, SOX2, and SSEA1, which is expected for bone fide iPS and ES cells (Figure 6). RT-PCR analysis confirmed reactivation of endogenous Myc, Oct 4 and Sox2 genes, as well as that of other pluripotency-associated transcription factors such as Ssea-1 and Nanog, observable in all iPS lines tested in the study (Figure 7A). The positive results of K13 tests showed there has some iPS cells came from counjunctival epithelial cells (Figure 7A).

We next evaluated the differentiation potential of the conjunctiva-iPS in vitro by embryoid body (EB) formation and in vivo by teratoma induction. The iPS cells readily formed EBs on culture in the absence of LIF and bFGF (Figure 4E). RT-PCR testing of the EBs showed strong suppression of the pluripotency gene $N A N O G$, with coincident activation of lineagespecific genes representing the three germ layers: nestin (ectoderm); vimentin and brachyury (mesoderm); and Foxa2 (endoderm) (Figure 7B). Each cell line was injected into three SCID mice. All 6 SCID mice formed teratomas. Hematoxylin-eosin staining of teratoma sections showed tissues representative of the three germ layers, including neuroepithelial cells, neuroepithelium with pigment (ectoderm), adipocytes, cartilage (mesoderm), and secreting-gland epithelium (endoderm) (Figure 8), providing strong evidence for the pluripotency of iPS cells generated with our methods. Normal karyotypes were achieved in both conjunctiva-induced iPS cell lines (Figure 9).

\section{Discussion}

The present study demonstrates the feasibility of generating induced pluripotent stem (iPS) cells from the conjunctiva. The reprogrammability of conjunctival cells was determined by various assays. RT-PCR analysis confirmed the presence of pluripotency-associated transcription factors. Pluripotency markers found in iPS derived from conjunctiva are identical to those found in ES lines. The differentiation potential of the conjunctiva-iPS was tested in vitro by EB formation, and in vivo by teratoma induction. This is the first demonstration that iPS can be isolated from the conjunctival cells, adding to previous studies of iPS differentiation showing that skin and liver cells, among others, are viable sources for reprogramming.

It remains to be determined whether iPS retains some of the original qualities of donor tissues [2]. When differentiated into ocular surface tissue, it may be that conjunctiva-iPS expresses ocular surface markers with greater intensity than would typical skin fibroblasts. Future studies may investigate whether conjunctiva-iPS is a particularly effective source of ocular surface tissue. If so, this would have encouraging implications for direct reprogramming.

The mice employed in the study express the four reprogramming genes under control of a doxycyline-inducible Collal promoter, R26rtTA; Colla14F2A [17]. Previously, R26rTA mice have been tested for inducible target gene expression in the liver, bone marrow, stomach, intestine, and skin, all with successful results. Low levels of reprogramming gene expression were discovered in the heart, lungs, kidney, spleen, and thymus; no expression was detected in the brain and testes [17]. The reprogramming efficiencies of conjunctiva iPS cells and ear fibroblasts cells are equivalent. Thus, expression of the four reprogramming genes Oct4, Sox2, Klf4, and Myc from the Collal promoter successfully yielded iPS from mouse conjunctival tissue. 
Routine ophthalmic procedures offer numerous opportunities to take samples of the conjunctiva [11, 18-20]. The anatomical location of the tissue makes it readily accessible and only a small sample may need to be taken to make iPS cells. Whenever small pieces of conjunctival tissue are taken during routine procedures, these may be placed in primary cell cultures and be kept for further use rather than discarded prematurely. Recently, it has been shown that dental pulp cells can also be used to create iPS cell banks [21], and we have demonstrated that it is possible to generate iPS from a similarly small type of biopsy. Conjunctival cells or, alternatively, conjunctiva-iPS cells, may be stored in a freezer bank for use in the future. Suitable candidates for conjunctival tissue sampling are wet AMD patients and diabetic patients who require ophthalmic procedures. These patients may benefit from iPS-based regenerative medicine treatments in the future.

\section{Acknowledgments}

Funding: This study was supported by NIH core grants 5P30CA013696 and P30EY019007, Research to Prevent Blindness (R01EY018213), the Research to Prevent Blindness Physician-Scientist Award, the Barbara and Donald Jonas Family Fund, the Schneeweiss Stem Cell Fund, New York State (N09G-302), and the Foundation Fighting Blindness New York Regional Research Center Grant (C-NY05-0705-0312).

SH Tsang is a Fellow of the Burroughs-Wellcome Program in Biomedical Sciences, and has been supported by the Bernard Becker Association of University Professors in Ophthalmology Research to Prevent Blindness Award, the Dennis W. Jahnigen Award of the American Geriatrics Society, the Joel Hoffman Fund, the Gale and Richard Siegel Stem Cell Fund, the Charles Culpeper Scholarship, the Irma T. Hirschl Charitable Trust, the Bernard and Anne Spitzer Stem Cell Fund, the Professor Gertrude Rothschild Stem Cell Foundation, and the Alan Gebroe Fund.

\section{References}

1. Li Y, Tsai YT, Hsu CW, Erol D, Yang J, Wu WH, Davis RJ, Egli D, Tsang SH. Long-term safety and efficacy of human-induced pluripotent stem cell (iPS) grafts in a preclinical model of retinitis pigmentosa. Mol Med. 2012; 18:1312-1319. [PubMed: 22895806]

2. Aoi T, Yae K, Nakagawa M, Ichisaka T, Okita T, Takahashi K, Chiba T, Yamanaka S. Generation of pluripotent stem cells from adult mouse liver and stomach cells. Science. 2008; 321:699-702. [PubMed: 18276851]

3. Stadtfeld M, Brennand K, Hochedlinger K. Reprogramming of pancreatic beta cells into induced pluripotent stem cells. Curr Biol. 2008; 18:890-894. [PubMed: 18501604]

4. Hanna J, Markoulaki S, Schorderet P, Carey BW, Beard C, Wernig M, Creyghton MP, Steine EJ, Cassady JP, Foreman R, Lengner CJ, Dausman JA, Jaenisch R. Direct reprogramming of terminally differentiated mature B lymphocytes to pluripotency. Cell. 2008; 133:250-264. [PubMed: 18423197]

5. Eminli S, Utikal J, Arnold K, Jaenisch R, Hochedlinger K. Reprogramming of neural progenitor cells into induced pluripotent stem cells in the absence of exogenous Sox 2 expression. Stem Cells. 2008; 26:2467-2474. [PubMed: 18635867]

6. Kim JB, Zaehres H, Wu G, Gentile L, Ko K, Sebastiano V, Araúzo-Bravo MJ, Ruau D, Han DW, Zenke M, Schöler HR. Pluripotent stem cells induced from adult neural stem cells by reprogramming with two factors. Nature. 2008; 454:646-650. [PubMed: 18594515]

7. Aasen T, Raya A, Barrero MJ, Garreta E, Consiglio A, Gonzalez F, Vassena R, Bilic J, Bekarik V, Tiscornia G, Edel M, Boue S, Izpisua Belmonte JC. Efficient and rapid generation of induced pluripotent stem cells from human keratinocytes. Nat Biotechnol. 2008; 26:1276-1284. [PubMed: 18931654]

8. Maherali N, Sridharan R, Xie W, Utikal J, Eminli S, Arnold K, Stadfeld M, Yachechko R, Tchieu Jaenisch R, Plath K, Hochedlinger K. Directly reprogrammed fibroblasts show global epigenetic remodeling and widespread tissue contribution. Cell Stem Cell. 2007; 1:55-70. [PubMed: 18371336]

9. Krause K, Foitzik K. Biology of the hair follicle: the basics. Semin Cutan Med Surg. 2006; 25:2-10. [PubMed: 16616298] 
10. Botchkarev VA, Paus R. Molecular biology of hair morphogenesis: development and cycling. J Exp Zool B Mol Dev Evol. 2003; 298:164-180. [PubMed: 12949776]

11. Mazow ML. The efficacy of conjunctival biopsy as a screening technique in lysosomal storage disorders. Trans Am Ophthalmol Soc. 1987; 85:471-497. [PubMed: 3328919]

12. Zinman HM, Joneja MG, Davies P, Smith BT. Cell culture of embryonic chick duodenal cells: preparation of epithelial-fibroblast bilayers and homotypic cultures of fibroblasts and epithelial cells. Journal of pediatric gastroenterology and nutrition. 1985; 4:107-117. [PubMed: 3981355]

13. Ramirez-Miranda A, Nakatsu MN, Zarei-Ghanavati S, Nguyen CV, Deng SX. Keratin 13 is a more specific marker of conjunctival epithelium than keratin 19. Mol Vis. 2011; 17:1652-1661. [PubMed: 21738394]

14. Merjava S, Brejchova K, Vernon A, Daniels JT, Jirsova K. Cytokeratin 8 is expressed in human corneoconjunctival epithelium, particularly in limbal epithelial cells. Investigative ophthalmology \& visual science. 2011; 52(2):787-794. [PubMed: 20926822]

15. Gilles C, Polette M, Zahm JM. Vimentin contributes to human mammary epithelial cell migration. J Cell Sci. 1999; 112(24):4615-4625. [PubMed: 10574710]

16. Thomson JA, Itskovitz-Eldor J, Shapiro SS, Waknitz MA, Swiergiel JJ, Marshall VS, Jones JM. Embryonic stem cell lines derived from human blastocysts. Science. 1998; 282:1145-1147. [PubMed: 9804556]

17. Carey BW, Markoulaki S, Beard C, Hanna J, Jaenisch R. Single-gene transgenic mouse strains for reprogramming adult somatic cells. Nat Methods. 2010; 7:56-59. [PubMed: 20010831]

18. Libert J, Van Hoof F, Tondeur M. Fucosidosis: ultrastructural study of conjunctiva and skin and enzyme analysis of tears. Invest Ophthalmol. 1976; 15:626-639. [PubMed: 955828]

19. Burns LR. Medical organization structures that promote quality and efficiency: past research and future considerations. Qual Manag Health Care. 1995; 3:10-18. [PubMed: 10144780]

20. Schmidt S, Hauser MA, Scott WK, Postel EA, Agarwal A, Gallins P, Wong F, Chen YS, Spencer K, Schnetz-Boutaud N, Hanies JL, Pericak-Vance MA. Cigarette smoking strongly modifies the association of LOC387715 and age-related macular degeneration. Am J Hum Genet. 2006; 78:852-864. [PubMed: 16642439]

21. Tamaoki N, Takahashi K, Tanaka T, Ichisaka T, Aoki H, Takeda-Kawaguchi T, Iida K, Kunisada T, Shibata T, Yamanaka S, Tezuka K. Dental pulp cells for induced pluripotent stem cell banking. J Dent Res. 2010; 89:773-778. [PubMed: 20554890] 


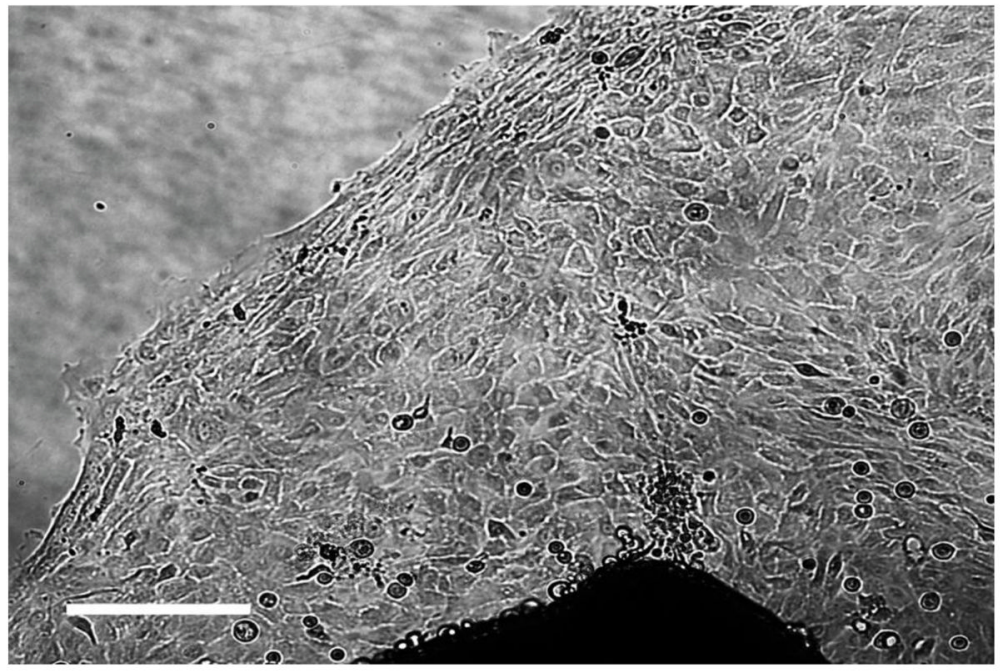

Fig. 1. Primary conjunctival cells cultured (P0) at day 3

Monolayer conjunctival cells grow out of conjunctival tissue at day 2 (20X). The primary epithelial cells have a cobblestone-like appearance. Scale bar: $100 \mu \mathrm{m}$ 


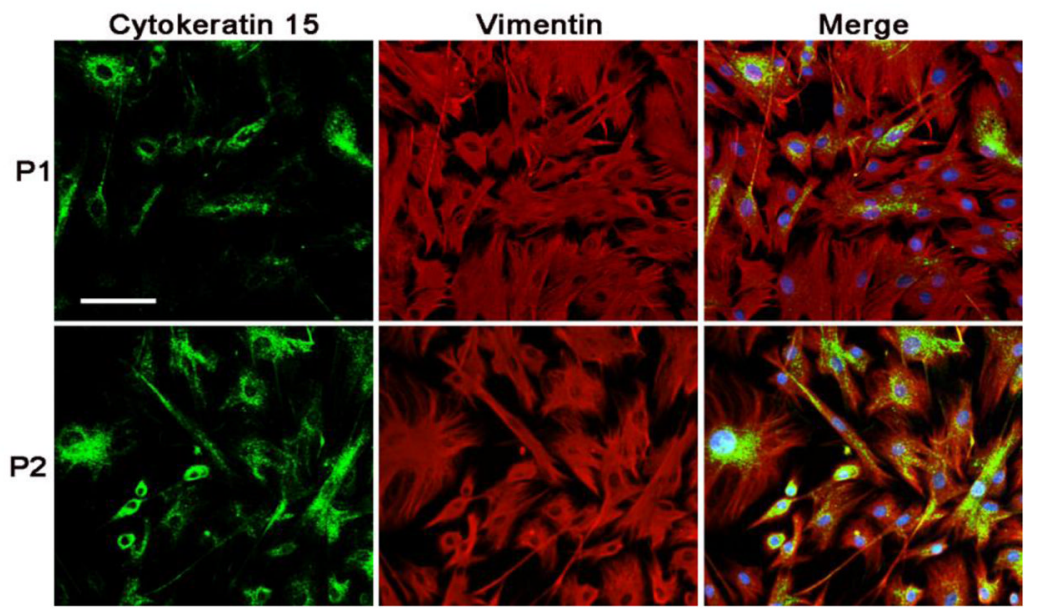

Fig. 2. Enrichment of conjunctival epithelial cells after collagenase IV treatment Cytokeratin 15(CK15) is the marker of conjunctiva epithelium cells. Vimentin expressed both on conjunctiva epithelium cells and fibroblasts. In the primary cell culture, the CK 15 positive epithelial cells occupied $30 \%$ of all attached cells (P1) and purified after collagenase IV 30 minutes administration which can float away most of fibroblasts(P2). (20x) Scale bar: $50 \mu \mathrm{m}$ 

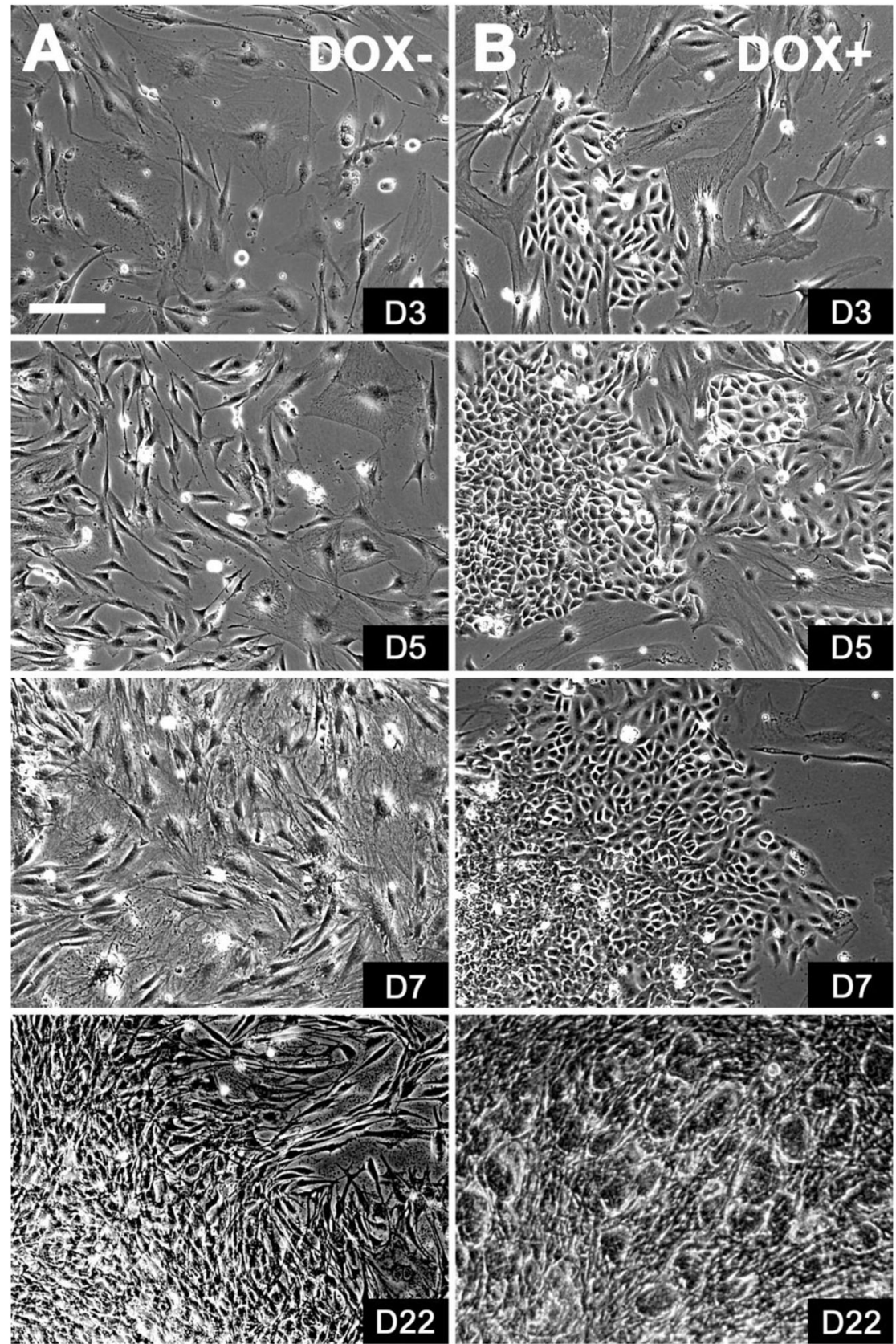

Fig. 3. iPS cells can be generated from conjunctival cells

Phase contrast imaging of cells demonstrates the compaction and transformation of conjunctival cells into iPS colonies, from day 3 (D3+dox) to day 12. Doxycyline (dox) was withdrawn on D12. A: Negative control image from dox- plate (20X). D5, D7: No changes were apparent in control untreated group except for cell growth; B: Morphological transformation of the mouse conjunctiva to the iPS state. Cells began to cluster during the first few days after dox treatment, and cells became rounder. Cells begin to exhibit a higher nucleus to cytoplasm ratio. The characteristic dome-shaped, refractile colonies of iPS cells appeared during day 20 and day 22; (20X) Scale bar: $100 \mu \mathrm{m}$. 

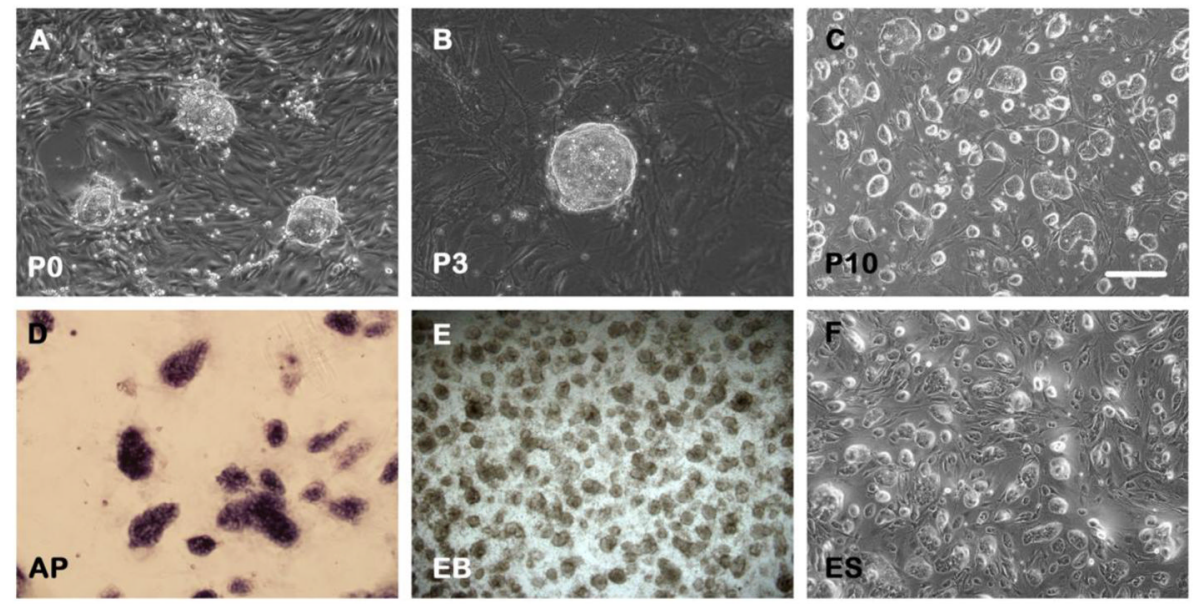

Fig. 4. iPS cells derived from the conjunctiva resemble ES cells

A, B, C: Representative images of colonies observed at different stages as indicated in the panels, from P0 (20X), to P3 (40X), to P10 (10X). A: The roundest and most evident border colonies were chosen to be manually picked. D: Alkaline phosphatase (AP) staining was positive (20X). E: In vitro differentiation of conjunctiva-iPS cells to embryonic bodies (4X). F: ES cells from the C57BL6/J mouse (10X). The morphology of conjunctiva iPS cells (C) was similar to that of ES cells $(\mathbf{F})$, which display a peripheral halo. Scale bar: $100 \mu \mathrm{m}$. 
A
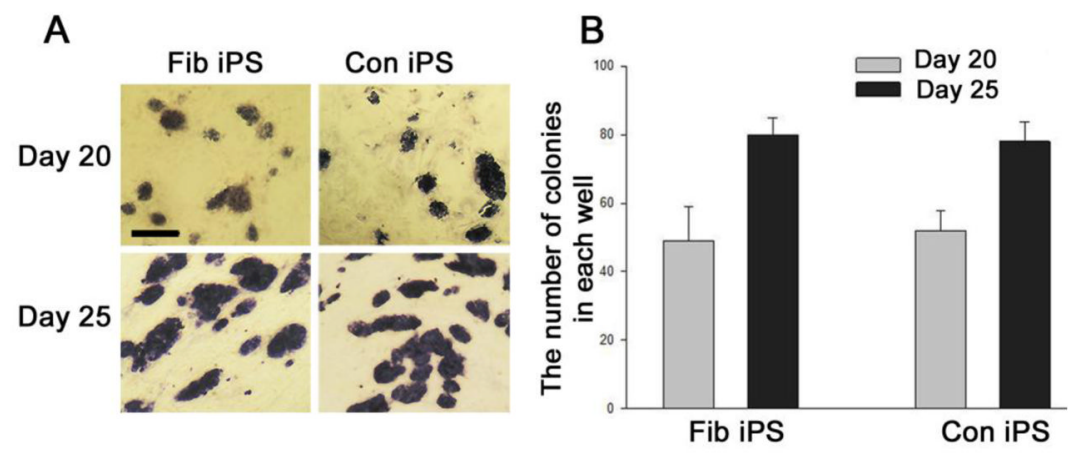

Fig. 5. The reprogramming efficiencies of conjunctiva iPS cells and ear fibroblasts cells are equivalent

A: AP staining of each cell line on day 20 and 25 . B: Total colony formation, as of days 20 and 22 , was measured and compared between dishes $(t=0.8581, \mathrm{p}>0.05)$. The reprogramming efficiency of the conjunctiva does not differ from that of ear fibroblasts. (Fib: fibroblast, Con: conjunctiva) (20X). Scale bar: $50 \mu \mathrm{m}$. 

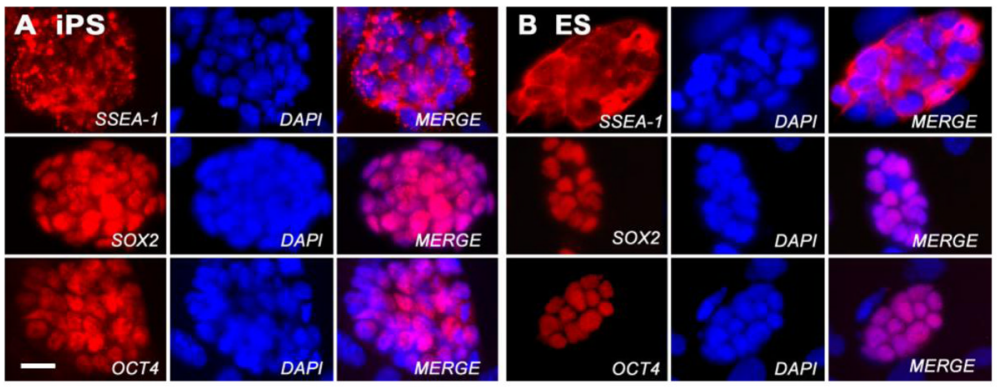

Fig. 6. A comparison of expression of pluripotency markers between iPS and ES cells A: Immunofluorescence staining shows expression of pluripotency markers (OCT4, SOX2, and SSEA1) in the dox-induced conjunctiva-iPS lines. B: These are compared to pluripotency markers in ES colonies. Middle columns show DAPI staining to visualize the nuclei. Rightmost panels show combined images of the stained nuclei with the stem cell markers in overlaid color. The iPS cells' pluripotency markers duplicate those of the ES cells. (40X). Scale bar: $25 \mu \mathrm{m}$. 


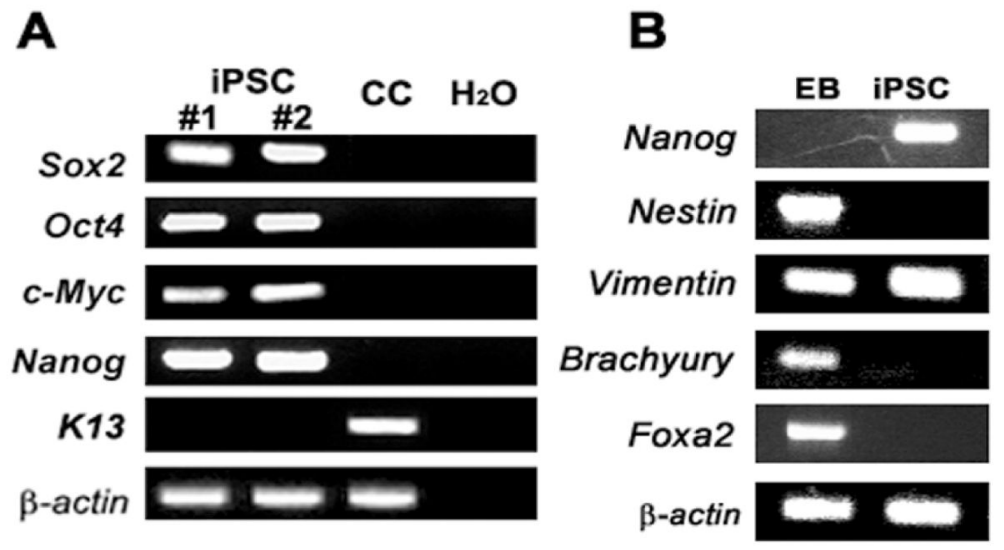

Fig. 7. Expression of pluripotency and differentiation markers by RT-PCR

A: RT-PCR analysis confirmed reactivation of endogenous c-Myc, Sox2, Oct4, K13 and Nanog. K13 is a specific maker for conjunctival epithelium. B: RT-PCR analysis of total RNA isolated from embryoid bodies (EBs) generated from conjunctival iPS lines. The expression of Nanog (a mark of pluripotency); vimentin and brachyury (mesoderm); Foxa2, (endoderm); and nestin (ectoderm) were examined. $\beta$-actin was used as a loading control. ( $\mathrm{CC}=$ conjunctival cells $)$. 

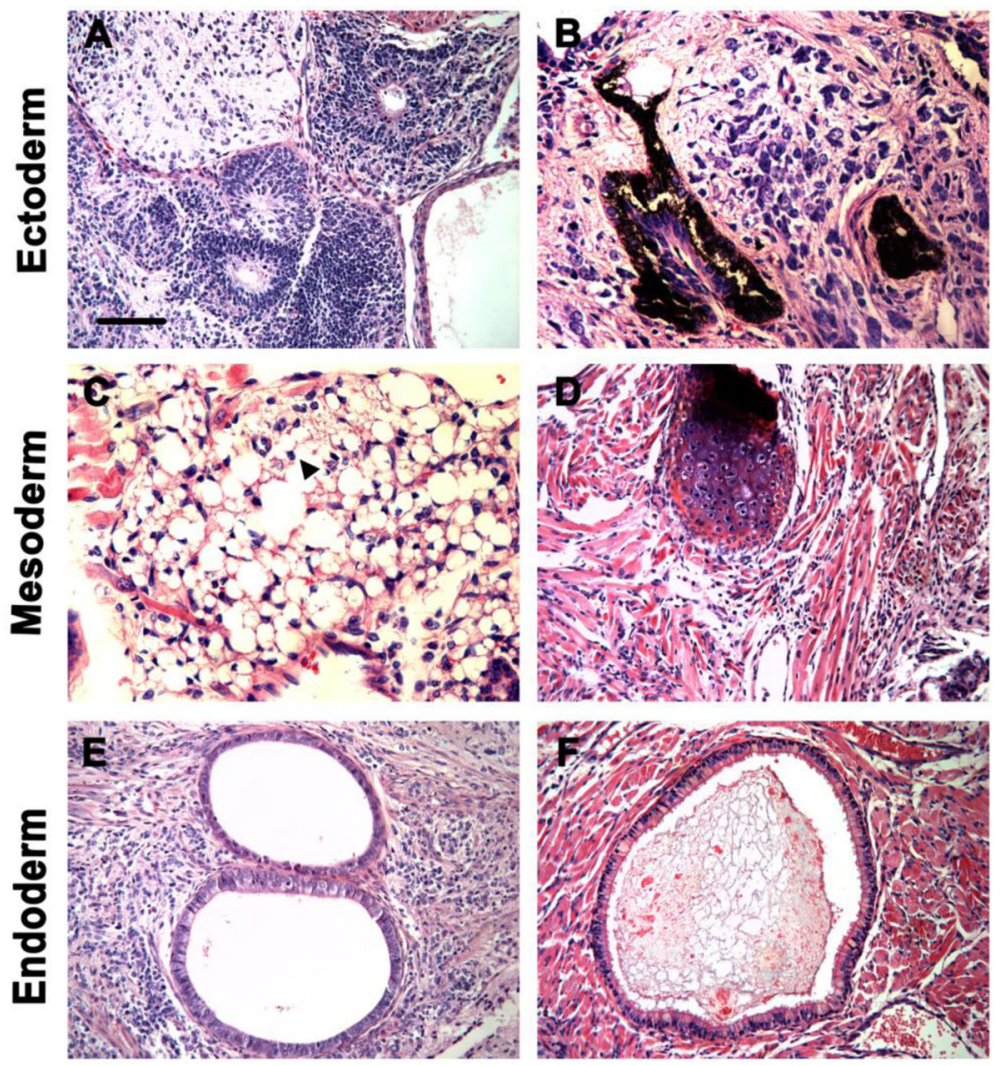

Fig. 8. iPS cells were differentiated into cell types belonging to all germ layers

After in vivo differentiation of conjunctiva-IPS, histologic analysis of teratomas revealed these three germ layers. A: Neuroepithelium showing stratified epithelium arranged in rosettes; B: Neuroepithelial cells with pigment (ectoderm); C: Variably sized individual immature adipocytes with centrally located nuclei (arrows); D: Cartilage (mesoderm), and E, F: Secreting-gland epithelium; Immature gland surrounded by immature smooth muscle (endoderm). Scale bar: $100 \mu \mathrm{m}$. 

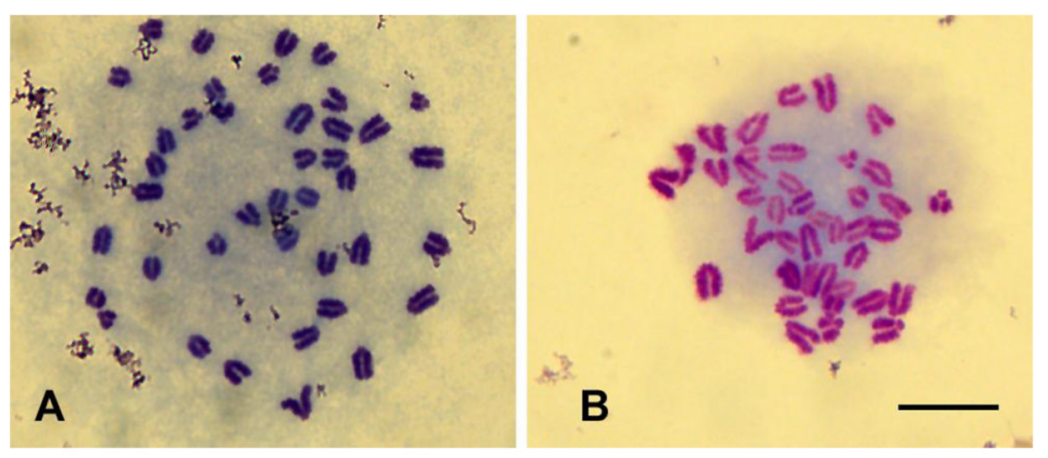

Fig. 9. Normal karyotype of the two conjunctiva-induced iPS cell lines

40 chromosomes were normal in two iPS cell lines derived from two different genetically modified mice (100x) (A and B were derived from these two mice). Scale bar: $25 \mu \mathrm{m}$. 


\section{Table}

Primer sequences

\begin{tabular}{lcr}
\hline Gene name & Forward primers $\left(\mathbf{5}^{\prime}\right.$ to $\left.\mathbf{3}^{\prime}\right)$ & Reverse primers $\left(\mathbf{5}^{\prime}\right.$ to $\left.\mathbf{3}^{\prime}\right)$ \\
\hline For RT-PCR & & \\
Endogenous & & \\
Sox-2 & TAGAGCTAGACTCCGGGCGATGA & TTGCCTTAAACAAGACCACGAAA \\
Nanog & TCAAGGACAGGTTTCAGAAGCA & GCTGGGATACTCCACTGGTG \\
Myc & TGACCTAACTCGAGGAGGAGCT & AAGTTTGAGGCAGTTAAAATTAT \\
Oct4 & TCTTTCCACCAGGCCCCCGGCTC & TGCGGGCGGACATGGGGAGATCC \\
K13 & CTGAACAAGGAGGTGTCTACCA & ATAGCGGCACTCCGTCTCT \\
$\beta$-actin & GGAATCCTGTGGCATCCATGAAAC & AAAACGCAGCTCAGTAACAGTCCG \\
For the three germ layer marker RT-PCR & \\
Differentiation markers & \\
Nestin & TCTGGAAGTCAACAGAGGTGG & ACGGAGTCTTGTTCACCTGC \\
Vimentin & GATGTTTCCAAGCCTGACCTC & GGCGTTCCAGAGACTCGTTAG \\
Brachyury & CATGTACTCTTTCTTGCTGG & GGTCTCGGGAAAGCAGTGGC \\
Foxa 2 & TGGTCACTGGGGACAAGGGAA & GCAACAACAGCAATAGAGAAC \\
\hline
\end{tabular}

Graefes Arch Clin Exp Ophthalmol. Author manuscript; available in PMC 2015 March 01. 